Markus Schwaninger/Christian Kaiser*

\title{
Erfolgsfaktoren organisatorischen Wandels ${ }^{* *}$
}

\section{Zusammenfassung}

Die empirische Forschung hat sich fast nicht mit der Frage nach den Erfolgsfaktoren von Projekten der organisatorischen Veränderung befasst. Um diese Lücke zu schließen, wurde eine exploratorische Studie mit einer Erhebung bei neun Großunternehmen durchgeführt. Aufgrund der Datenanalyse wurden die für den Projekterfolg maßgeblichen Faktoren eruiert. Es handelt sich dabei um das Engagement der Entscheidungsträger, die sachliche Ausrichtung der Entscheidungen, die Klarheit der Ziele, Partizipation der Betroffenen und einen ganzheitlichen Ansatz der Projektrealisierung. Diese Ergebnisse wurden in den Tests bestätigt, sind also als robust zu betrachten.

JEL-Classification: M10.

Keywords: Change Projects; Critical Success Factors; Organizational Change; Organizational Projects.

Kritische Erfolgsfaktoren; Organisationsprojekte; Organisatorischer Wandel; Veränderungsprojekte.

\section{Hintergrund/Einleitung}

Der organisatorische Wandel ist ein in der Fachliteratur über Management und Organisation vieldiskutiertes Thema. Nicht nur theoretisch-konzeptionelle Abhandlungen, sondern auch pragmatische Handlungsempfehlungen an die Adresse von Führungskräften werden häufig formuliert. Trotzdem besteht auf diesem Gebiet noch erheblicher Orientierungsund Forschungsbedarf.

* Professor Dr. Markus Schwaninger, Universität St. Gallen, IfB-HSG, Institut für Betriebswirtschaft an der Universität St. Gallen, Dufourstr. 40a, CH-9000 St. Gallen; Dr. Christian Kaiser, FEW-HSG Forschungsinstitut für Empirische Ökonomie und Wirtschaftspolitik an der Universität St. Gallen und ZBM-HSG Zentrum für Business Metrics, Varnbüelstr. 14, CH-9000 St. Gallen.

** Die Autoren danken Herrn Dr. Sebastian Raisch und einem anonymen Gutachter für wertvolle Anregungen. 
Aus diesem Grunde wurde am Institut für Betriebswirtschaft der Universität St. Gallen ein Kompetenzzentrum für Systemische Projektsteuerung gegründet, zwecks Realisierung von Erfahrungsaustausch sowie gemeinsamer Forschungs- und Entwicklungsaktivitäten. Bei den in der ersten Etappe mitwirkenden acht Partnerfirmen - durchwegs mittelgroße bis große Unternehmungen - zeigte sich bald ein Interesse an den Erfolgsfaktoren von Organisations- und Veränderungsprojekten. Dieses Thema speziell zu untersuchen, sollte nicht zuletzt deshalb lohnend sein, weil die Erfolgsfaktoren von Projekten in Abhängigkeit vom Projekttyp variieren können ${ }^{1}$. Dies impliziert, dass wahrscheinlich bei Organisationsprojekten andere Erfolgsfaktoren auftreten als beispielsweise in Entwicklungs- oder Bauprojekten.

Eine Sichtung der Literatur zeigte, dass eine Forschungslücke besteht. Bezüglich Erfolgsfaktoren von Organisations- und Veränderungsprojekten gibt es bisher fast keine empirischen Studien. Eine Ausnahme bildet ein Text über Erfolgsfaktoren beim Business Process Reengineering; dessen Beschränkung ist allerdings, dass er auf nur einer Fallstudie basiert ${ }^{2}$. Zwei weitere Untersuchungen mit Umfragen bei Projektmanagern und -mitarbeitern stellt zwar die Frage nach den kritischen Erfolgsfaktoren ${ }^{3}$ respektive allgemein nach den Erfolgsfaktoren bei Projekten ${ }^{4}$, es erfolgt dort aber keine Auswertung nach Arten von Projekten, das heißt, die Analyse gibt keine Anhaltspunkte, welche der Faktoren speziell für Organisations- und Veränderungsprojekte von Bedeutung ist. Ebenfalls nicht nach Projekttyp unterschieden wird in einer Reihe von Untersuchungen, die jeweils Teilaspekten gewidmet sind, etwa der Implementierung bei Bau- sowie Forschungs- und Entwicklungsprojekten ${ }^{5}$, den Gründen von Projektmisserfolg ${ }^{6}$ oder strukturellen Gründen für den Erfolg von Projekten ${ }^{7}$.

Das Thema der Erfolgsfaktoren von Organisations- und Veränderungsprojekten wurde am genannten Kompetenzzentrum in Workshops und qualitativen Interviews mit den Unternehmungsvertretern explorativ bearbeitet. Dabei wurden Beurteilungen der Managementsysteme für Organisations- und Veränderungsprojekte vorgenommen und Kandidaten für Erfolgsfaktoren ermittelt. Darüber hinaus wurde der Beschluss gefasst, eine Befragung durchzuführen, um die empirische Basis zu verbreitern.

Ziel des vorliegenden Artikels ist es, die Ergebnisse dieser Befragung darzustellen und zu diskutieren. In Abschnitt 2 werden Zielsetzung und Methodik der Studie dargelegt. Konzeptionelle Grundlagen bezüglich der Erfolgsfaktoren organisatischen Wandels enthält Abschnitt 3. In den Abschnitten 4 und 5 werden Design und Abwicklung der Befragung dokumentiert. Die Ergebnisse der Datenanalyse sind in Abschnitt 5 dargelegt. Abschließend wird ein Fazit gezogen, in dem die Resultate der Studie mit ihren Implikationen zusammenfasst werden (Abschnitt 7).

1 Vgl. Dvir et al. (1998).

2 Paper/Chang (2005).

3 Dvir et al. (2003).

4 Cremer (2002).

5 Zum Beispiel Pinto/Covin (1989).

6 Pinto/Mantel (1990).

7 Might/Fisher (1985). 


\section{Zielsetzung und Methodik}

Diese Untersuchung analysiert die Erfolgsfaktoren von Organisations- und Veränderungsprojekten. Die zu beantwortende Forschungsfrage lautet: Welche Faktoren beeinflussen den Erfolg von Organisations- und Veränderungsprojekten signifikant? Ergänzend wird die Frage gestellt: Bestehen Unterschiede bezüglich der Wirkung dieser Einflussgrößen auf verschiedene Erfolgsmaßstäbe?

Es handelt sich um eine explorative, nicht um eine hypothesentestende Studie. Zwecks Erzielung möglichst allgemeingültiger Ergebnisse wurde das Instrument der Befragung einer größeren Stichprobe eingesetzt. Befragt wurden Führungskräfte mit Projektleitungserfahrung und Projektmitarbeiter in den acht Unternehmungen, die am Kompetenzzentrum Systemische Projektsteuerung mitwirkten. Je Branche war plangemäß nur ein Unternehmen in dieser Plattform vertreten, um Konkurrenzprobleme auszuschalten. Somit handelte es sich um Unternehmungen aus acht verschiedenen Branchen (Tabelle 1).

\section{Theoretisch-konzeptionelle Grundlagen}

Vorerst gilt es, die beiden Komponenten des Untersuchungstitels - Erfolgsfaktoren und organisatorischer Wandel - theoretisch-konzeptionell zu beleuchten.

Unter einem Erfolgsfaktor wird eine Einflussgröße verstanden, die mit dem Erfolg einer Unternehmung korreliert ist, so dass ein kausaler Einfluss vermutet, wenn auch nicht bewiesen werden kann. Das Ausmaß der Korrelation kann variieren. Im Falle sehr hoher Korrelation wird auch von kritischen Erfolgsfaktoren gesprochen. Die Erfolgsfaktorenforschung hat eine lange Tradition ${ }^{8}$. Von den vielen Studien, die auf diesem Gebiet realisiert worden sind, ist ein widerspruchsfreier Satz von Ergebnissen bislang nicht ermittelt worden. Es gibt Kritik an der Erfolgsfaktorenforschung; diese geht so weit, dass sogar behauptet wird, es gebe „derzeit kein einziges Ergebnis aus der PaaV [Performance als abhängige Variable]-Forschung, das als gesichert gilt" ${ }^{\text {“9. }}$. Dies zeigt aber gerade, dass weiterer Forschungsbedarf unter Berücksichtigung kritischer Punkte dringend nötig ist. Unsere Studie ist deshalb von hohem Interesse.

Nicolai und Kieser (2002) führen zahlreiche weitere Einwände gegen die Erforschung von Erfolgsfaktoren an, insbesondere die Uneinheitlichkeit ihrer Ergebnisse. Diese Argumente sind durch Entgegnungen relativiert worden. Beispielsweise ist die Erfolgsfaktorenforschung zu einer Reihe von übereinstimmenden Ergebnissen gekommen ${ }^{10}$. Zudem ist sie nicht als ein Forschungsgebiet zu betrachten, dem nichts mehr hinzuzufügen wäre, sondern sie ist als potentiell fortschrittsfähig zu bezeichnen ${ }^{11}$.

8 Vgl. Rockart (1979); Leidecker/Bruno (1984); Hildebrandt (1992); Lehner (1995).

9 Nicolai/Kieser (2002), S. 582.

10 Fritz (2004).

11 Bauer/Sauer (2004). 
Die Kritik von Kieser und Nicolai bezieht sich übrigens auf Studien, in denen finanzwirtschaftliche Größen wie ROI, ROE, ROA, ROS ${ }^{12}$ oder Earnings per Share die abhängigen Variablen bilden ${ }^{13}$. Wir betrachten im Gegensatz dazu nicht-finanzwirtschaftliche Erfolgsgrößen, eine davon (im Folgenden als $f 28$ bezeichnet) als vieldimensionales Konstrukt.

Für eine solche Studie ist es wichtig, Organisations- und Veränderungsprojekte (OUV) von anderen Projekttypen zu unterscheiden, weil davon auszugehen ist, dass sich die Erfolgsfaktoren der Typen wesentlich unterscheiden ${ }^{14}$.

OUV sind zeitlich befristete, zielorientierte Vorhaben mit in der Regel innovativem Charakter, an denen verschiedene - oft viele - Personen aus unterschiedlichen Organisationseinheiten und Disziplinen mitwirken ${ }^{15}$. Umfang und Komplexität solcher Vorhaben sind in der Regel hoch.

Im Gegensatz zu anderen Arten von Projekten sind OUV - im hier verstandenen Sinn - Reorganisationen, also planvolle, tiefgreifende Umgestaltungen von Unternehmungen oder Teilen davon ${ }^{16}$. Im Vergleich zu OUV sind Entwicklungsprojekte, Informatikprojekte, Bauprojekte usw. tendenziell stärker standardisierbar und hinsichtlich ihres Wirkungsradius weniger umfangreich. Auch sind ihre Umweltcharakteristika im Prinzip anders ${ }^{17}$. Aus all diesen Gründen beschränkt sich diese Studie auf OUV.

\section{Design der Befragung}

Das Thema Organisations- und Veränderungsprojekte ist empirisch erst wenig erschlossen. Zudem sollte es in diesem Fall nicht zu eng betrachtet werden. Deshalb der explorative Charakter der Befragung. Wir legen uns bewusst nicht auf einige wenige, detaillierte Hypothesen fest, sondern streben ein eher breites Bild an, das Aufschluss über eine Reihe von Erscheinungen, Trends und Zusammenhängen gibt. Die Daten werden statistisch analysiert und ermöglichen damit, empirisch fundierte Antworten zur Fragestellung betreffend Erfolgsfaktoren von OUV zu geben.

Der Fragebogen umfasst drei Gruppen von Fragen, folgende Untersuchungsebenen betreffend:

1. Generelle Entwicklungen auf dem Gebiet der OUV (5 Fragen)

2. OUV in der Unternehmung der befragten Person (5 Fragen)

3. Ein konkretes, gut eingrenzbares Projekt (34 Fragen für Projektleiter, 25 für Projektmitarbeiter)

12 Die Abkürzungen stehen für Return on Investment, Return on Equity, Return on Assets, Return on Sales.

13 Nicolai/Kieser (2002), S. 581.

14 Vgl. Haenecke (2002).

15 Vgl. Marr/Steiner (2004); Schwaninger/Körner (2003).

16 Gassner (1999); Picot/Freudenberg/Gassner (1999).

17 Schwaninger/Körner (2004), S. 97. 
Die auf Erfolgsfaktoren von OUV bezogenen Fragen sind weitestgehend auf den dritten Fragenblock konzentriert. Die Details zu den Fragen sind bei Schwaninger/Kaiser (2006) dokumentiert. Von den Fragen wurden für diese spezifische Untersuchung theoriegeleitet 14 ausgewählt. Diese Variablenliste ist im Wesentlichen das Ergebnis der Auswertung der im Vorfeld durchgeführten, explorativen Befragung (35 Interviews) in den beteiligten Unternehmungen. Im Folgenden werden die entsprechenden Variablen synoptisch dargestellt, strukturiert und begründet.

\section{Abbildung 1: Ausgewählte unabhängige und abhängige Variablen}

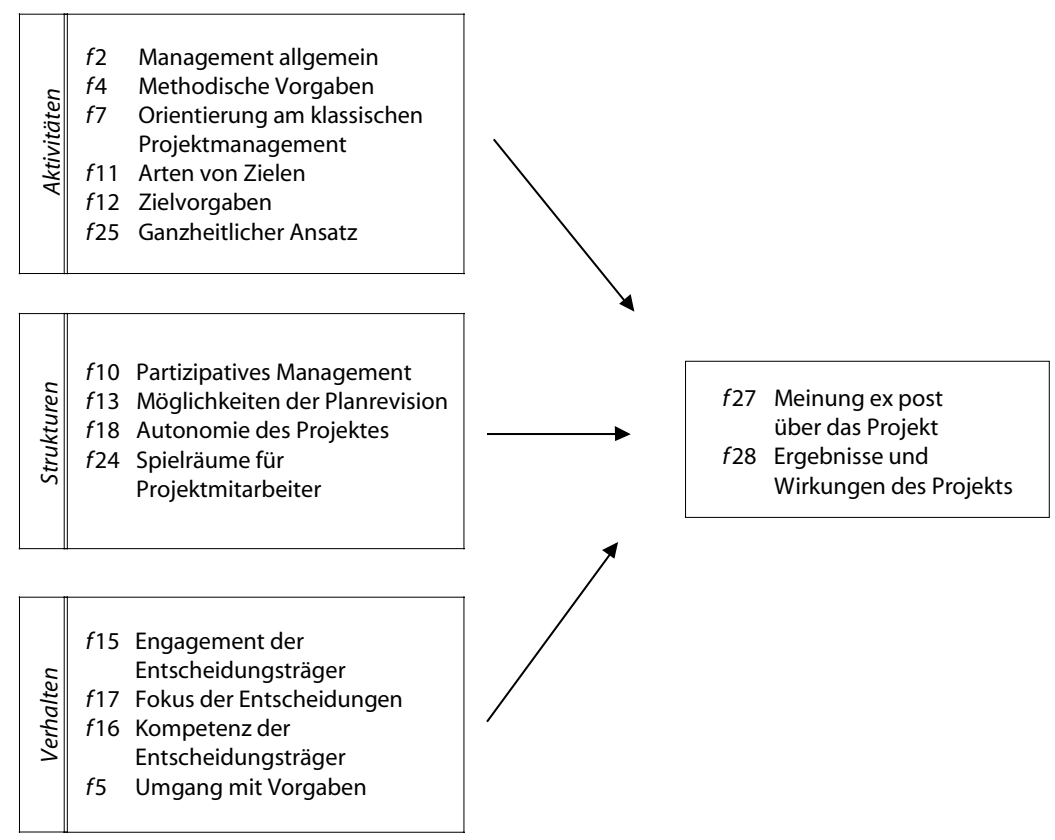

Die Darstellung in Abbildung 1 zeigt die unabhängigen und abhängigen Variablen im Zusammenhang. Der Satz unabhängiger Variablen bringt eine wichtige Unterscheidung zum Ausdruck. Bei breit angelegten Untersuchungen auf dem Gebiet des allgemeinen Managements sollten im Prinzip sowohl aktivitäts-, als auch struktur- und verhaltensbezogene Aspekte berücksichtigt werden ${ }^{18}$. Deshalb erfolgt die Gliederung der unabhängigen Variablen nach Aktivitäten, Strukturen und Verhalten, welche im Übrigen auch einer klassischen systemtheoretischen Kategorisierung entspricht ${ }^{19}$. 
Das Messmodell beruht auf ordinalen Messskalen, denn die Antworten verkörperten durchwegs Einschätzungen der Befragten. Bei Nichtverfügbarkeit „objektiver“ Daten, muss notwendigerweise auf subjektive Beurteilungen zurückgegriffen werden. Dass dieses, aufgrund eines Sachzwangs gewählte Vorgehen auch zuverlässig ist, wird durch empirische Evidenz untermauert. Beispielsweise stellten Robinson und Dess (1984) fest, dass die subjektiven Wahrnehmungen von Führungskräften, bezogen auf die durch Ihre Unternehmungen erzielten Ergebnisse, mit den entsprechenden objektiven Messgrößen stark korreliert waren.

Die Auswahl der Variablen erfolgte aufgrund des Studiums der einschlägigen Literatur. Von den unabhängigen Variablen, welche sich auf Aktivitäten des Projektmanagements beziehen, wurden sechs als potentielle Erfolgsfaktoren identifiziert.

- Management allgemein: Es wird unterstellt, dass der Rückgriff auf eine umfassende Methodik erfolgsträchtiger ist, als ein punktuelles Management ad hoc ${ }^{20}$.

- Methodische Vorgaben: Ähnlich wird davon ausgegangen, dass sich methodische Vorgaben tendenziell erfolgssteigernd auswirken ${ }^{21}$.

- Orientierung am klassischen Projektmanagement: Angenommen wird, dass Instrumente des klassischen Projektmanagements, wie beispielsweise MS-Project ${ }^{22}$, für Organisations- und Veränderungsprojekte wenig Mehrwert bringen ${ }^{23}$. Dies, weil es oft mit der sich in Prozessen des Wandels zeigenden Komplexität überfordert ist ${ }^{24}$.

- Arten von Zielen: Eine Beschränkung auf nur kurzfristige oder auf nur langfristige Zielsetzungen wurde für unzulänglich erachtet. Kurz- und langfristige Ziele gleichermaßen zu berücksichtigen, wird als erfolgsträchtiger beurteilt ${ }^{25}$.

- Zielvorgaben: Sowohl Klarheit als auch Verbindlichkeit von Zielvorgaben werden als Erfolgsfaktoren betrachtet, im Gegensatz zu unklaren, wenig verbindlichen Zielen ${ }^{26}$.

- Ganzheitlicher Ansatz: Ein ganzheitlicher Ansatz, der über einzelne, technische Funktionen und Themen hinausgeht und diese bewusst integriert, wird für eine erfolgreiche Projektrealisierung als wesentlich betrachtet ${ }^{27}$.

Von den unabhängigen Variablen, welche sich auf Strukturen des Projektmanagements beziehen, wurden die folgenden vier als potentielle Erfolgsfaktoren identifiziert:

- Partizipatives Management: Für den Erfolg eines Projekts müsste die aktive Mitwirkung der wichtigsten vom Projekt betroffenen Gruppen bedeutend sein. Die empirisch fundierte Literatur bestätigt zwar nicht einen generell positiven Zusammenhang

20 Siehe Ulrich (2001); vgl. Feldman/Pentland (2003).

21 Vgl. Schwaninger/Körner (2003); Schmidt (2003).

22 MS Project ist eine weit verbreitete, von der Firma Microsoft hergestellte Software für das Planen, Verwalten und Kommunizieren in Projekten.

23 Vgl. Williams (1999).

24 Schwaninger/Körner (2004).

25 Vgl. Gälweiler (2005); Schwaninger (1989).

26 Vgl. Drucker (1974), S. 82ff.

27 Vgl. Schwaninger (1984); Malik (1990). 
von Partizipation und Produktivität, sondern dieser gilt als situationsabhängig ${ }^{28}$; unter Abstraktion von möglichen intervenierenden Variablen ${ }^{29}$ und angesichts der Konzentration des Partizipationsprinzips auf die wichtigsten vom Projekt betroffenen Gruppen kann hier aber vorerst davon ausgegangen werden, dass Partizipation potentiell ein Erfolgsfaktor ist ${ }^{30}$.

- Möglichkeiten der Planrevision: Im Gegensatz zu einer Projektplanung als einmaliger Wurf sollten im Projektverlauf Gelegenheiten bestehen, die Planung zu überprüfen und zu verändern. Das bewährte Prinzip einer Planung als kontinuierlicher, iterativer und rekursiver Prozess ${ }^{31}$ stützt diese Annahme.

- Autonomie des Projektes: Relativ hohe, wenn auch nicht unbeschränkte, Autonomie ist generell als ein die Effektivität von Projektteams erhöhender Faktor empirisch untermauert ${ }^{32}$.

- Spielräume für Projektmitarbeiter: Hierbei geht es ähnlich wie bei der vorhergehenden Variablen um Autonomie, allerdings auf einer nächsttieferen Ebene - innerhalb des Projektteams. Da Handlungsspielräume eine Quelle der Macht sind ${ }^{33}$ und die intrinsische Motivation tendenziell fördern ${ }^{34}$, ist diese Variable analog der vorherigen zu interpretieren.

Von denjenigen unabhängigen Variablen, welche sich auf Verhaltensaspekte des Projektmanagements beziehen, wurden ebenfalls vier als potentielle Erfolgsfaktoren identifiziert.

- Engagement der Entscheidungsträger: Diese Größe wurde als Approximation für Energie verwendet, die bei Bruch (2003) als Treiber wirksamen Handelns figuriert. Deshalb wird sie hier als potentieller Erfolgsfaktor gesetzt.

- Fokus der Entscheidungen: Da nicht untersucht werden konnte, wie fokussiert die Projektleiter im Einzelnen tatsächlich vorgingen, wurde der Fokus der Entscheidungen in den Blickpunkt gerückt. Es wird davon ausgegangen, dass Entscheidungen, die überwiegend sachlich bedingt sind, solchen, die weitgehend interessenbedingt gefällt werden, bezüglich ihrer Erfolgswirkungen überlegen sind. Diese Annahme wird gestützt durch das Konzept der Instrumentalität nach Vroom (1964) ${ }^{35}$.

- Kompetenz der Entscheidungsträger: In der Strategieforschung wurde erhärtet, dass die Fähigkeiten von Managern maßgeblich für die in Organisationen erzielten Resultate $\operatorname{sind}^{36}$. Deshalb wird davon ausgegangen, dass die Kompetenz von Entscheidungsträgern erfolgskritisch ist.

28 Vgl. Bass (1990); Kirsch/Esser/Gabele (1979), S. 298ff. Zudem wurde eine praktisch insignifikante, trotz der im Allgemeinen theoretisch signifikanten, Produktivitätswirkung von Partizipation vermutet (Wagner (1994)).

$29 \mathrm{Vgl}$. Lam/Chen/Schaubroeck (2002).

30 Siehe auch die Befunde bei Schwaninger (1984); Lawler (1986); Malik (1990); Freudenberg (1999).

31 Vgl. Beer (1979); Schwaninger (1989).

32 Siehe z.B. Seibert/Seth/Randolph (2004).

33 Picot/Freudenberg/Gasser (1999).

34 Deci/Ryan (1993).

35 Gemäß Vroom (1964) konzentriert der Mensch seine Anstrengungen auf die Erreichung eines Zieles, wenn die Erreichung des Zieles von der Erbringung der eigenen Leistung abhängt (vgl. Lattmann (1977)).

36 Adner/Helfat (2003); Hjelt (2004). 
- Umgang mit Vorgaben: Latham und Kinne (1974) zeigten experimentell, dass ein Operieren unter maßvoll schwierigen Produktionszielen zu besseren Ergebnissen führt als in Kontrollgruppen, die solcher Zielsetzungen entbehren ${ }^{37}$. In Ableitung davon wird in dieser Studie angenommen, dass eine Beachtung von methodischen Vorgaben erfolgsträchtiger ist als ein Ignorieren derselben.

Was die abhängigen Variablen anbelangt, mit denen der Projekterfolg gemessen wird, beschränken wir uns auf zwei Messgrößen:

- die Einschätzung der Meinung, welche sich in der Unternehmung über das Projekt gebildet hat (dreistufige Skala von überwiegend positiv bis überwiegend negativ),

- die Beurteilung der Ergebnisse und Wirkungen des Projektes (14 Items ${ }^{38}$, z.B.: „Das Projekt hat ... seine vorgegebenen Ziele erreicht, ... die Umsätze erhöht, ... die Entwicklung des Unternehmens gefördert." Die Skala ist vierstufig, von trifft vollständig $z u$ bis trifft gar nicht $z u$ ).

\section{Vorbereitung und Durchführung der Befragung}

Die beiden Leiter des Kompetenzzentrums erarbeiteten - auf Basis der im Vorfeld geführten, explorativen Interviews (siehe oben) - zwei Fragebögen für die beiden Zielgruppen „Führungskräfte mit Projektleitungserfahrung“ und „Projektmitarbeiter“. Die meisten Fragen waren deckungsgleich. Anschließend wurden die ausgearbeiteten Fragebögen zur Expertenvalidierung fünf Fachleuten unterbreitet, die entweder Spezialisten für empirische Sozialforschung waren oder bereits Umfragen in verwandten Gebieten durchgeführt hatten. Nach der Berücksichtigung der Anregungen dieser fünf Experten wurden die fertigen Fragebögen bei 16 Unternehmensvertretern während des zweiten Workshops des Kompetenzzentrums im April 2004 getestet. Es zeigte sich, dass die Fragebögen problemlos von den Probanden verstanden und ausgefüllt wurden; die Ausfülldauer lag zwischen 9 und 18 Minuten.

Es resultierten nur noch marginale Anregungen für Verbesserungen des Instruments. Die eigentliche Befragung wurde mit Hilfe der Teleform-Software mittels elektronischer Fragebögen via Web durchgeführt. Wir vereinbarten am genannten Workshop mit den Unternehmensvertretern, dass sie die Fragebögen intern, möglichst an alle potentiellen Träger von für die Befragung relevantem Wissen verteilen würden. Davon versprachen wir uns im Vergleich zu einem streng nach Zufallsauswahl versandten Fragebogen a) eine höhere Kompetenz der Befragten für die Beantwortung der gestellten Fragen und b) eine höhere Rücklaufquote, da die Verteilung des Fragebogens durch firmeninterne Bezugspersonen dem Anliegen der Befragung innerhalb der Unternehmen mehr Nachdruck verleiht.

Die ausgefüllten Fragebögen wurden durch die Befragten per Knopfdruck an einen Server der Universität St. Gallen gesandt und dort ausgewertet. Insgesamt beträgt die durch-

37 Vgl. O'Leary-Kelly/Martocchio/Frink (1994).

38 Die Items wurden deduktiv ermittelt. 
schnittliche Rücklaufquote 41 Prozent (181 ausgefüllte von 443 verteilten Fragebögen; vgl. Tabelle 1). Dieser Wert darf als relativ hoch betrachtet werden. Bei Umfragen ohne Zusatzmaßnahmen werden heute nämlich selten Rücklaufquoten über $20 \%$ erzielt; je nach Zielgruppe kann häufig nur mit Quoten um die 5\% gerechnet werden ${ }^{39}$.

\section{Tabelle 1: Übersicht - Beteiligte Unternehmen und Rücklauf der Fragebögen}

\begin{tabular}{|c|c|c|c|c|}
\hline Branche & $\begin{array}{l}\text { Anzahl } \\
\text { Antworten } \\
\text { (Projekt- } \\
\text { mitarbeiter) }\end{array}$ & $\begin{array}{l}\text { Anzahl } \\
\text { Antworten } \\
\text { (Projektleiter) }\end{array}$ & $\begin{array}{l}\text { Anzahl } \\
\text { Befragte } \\
\text { insgesamt }\end{array}$ & $\begin{array}{l}\text { Anzahl } \\
\text { Antworten } \\
\text { insgesamt }\end{array}$ \\
\hline Beratung & 0 & 1 & 1 & 1 \\
\hline Informatik & 13 & 71 & 200 & 84 \\
\hline Bank & 12 & 25 & 100 & 37 \\
\hline Verpackungsindustrie & 7 & 16 & 60 & 23 \\
\hline Pharmaindustrie & 0 & 2 & 2 & 2 \\
\hline Maschinenindustrie & 2 & 5 & 25 & 7 \\
\hline Finanzdienstleistungen & 7 & 6 & 30 & 13 \\
\hline Zementindustrie & 3 & 1 & 15 & 4 \\
\hline Treuhand $^{40}$ & 0 & 10 & 10 & 10 \\
\hline TOTAL & 44 & 137 & 443 & 181 \\
\hline
\end{tabular}

\section{Datenanalyse}

Im Folgenden werden, ausgehend von einer Umschreibung der Datenbasis, zwei Stufen der Datenanalyse geschildert, zunächst eine deskriptive Analyse, dann Regressionsschätzungen.

\subsection{Datenbasis und deskriptive Analyse}

Wie in Abschnitt 3 erwähnt, wurde die Befragung für zwei Zielgruppen durchgeführt. Bei der ersten handelt es sich um Führungskräfte, bei der zweiten um Projektmitarbeiter. Die

39 Diekmann (1998), S. 441.

40 Die Treuhandunternehmung war nicht Teil des Kompetenzzentrums. Sie war aber interessiert, an der Befragung mit einer kleinen Anzahl Befragter teilzunehmen, was ihr ermöglicht wurde. 
hier dargestellten Ergebnisse der Datenanalysen ${ }^{41}$ beziehen sich generell auf die Projektleiter (PL-Stichprobe), da für sie die größere Anzahl an Beobachtungen (137) vorliegt. Vergleichbare Analysen mit den Daten für Projektmitarbeiter (PM-Stichprobe, $44 \mathrm{Beob}-$ achtungen) führen grundsätzlich zu ähnlichen Ergebnissen. Unterschiede werden im Text erwähnt.

Ziel der Untersuchung ist es, die Determinanten des Projekterfolgs im Sinne der Projektzufriedenheit zu bestimmen. Die Projektzufriedenheit lässt sich aus der Befragung anhand von zwei (abhängigen) Variablen erkennen. Bei der ersten (Frage 27 bzw. Variable f27) handelt es sich um die Antwort auf die Frage nach der allgemeinen Zufriedenheit nach Abschluss eines Projekts. Die Variable hat drei Ausprägungen ( $1=$,überwiegend positiv“, 2 = „Zweigeteilt positiv und negativ“, 3 = „überwiegend negativ"). ${ }^{42}$ Es zeigte sich, dass die Projektleiter den Projektausgang in fast $42 \%$ der Fälle überwiegend positiv und nur in $8,5 \%$ überwiegend negativ beurteilten. Bei den Projektmitarbeitern war die Zufriedenheit etwas geringer: $31 \%$ der Beurteilungen waren positiv und knapp 17\% negativ ${ }^{43}$.

Die zweite Variable, aus der die Projektzufriedenheit erkennbar ist, resultiert aus den Antworten auf Frage 28 (Variable $f 28$ ). Diese Frage beinhaltet 14 Teilfragen, in denen nach einer Beurteilung der positiven Ergebnisse des Projekts gefragt wird. Aus diesen wurde ein Indikator gebildet, indem aus den Teilfragen, die vom jeweiligen Teilnehmer beantwortet wurden, der Mittelwert gebildet wurde. Es stellt sich hier die Frage, wie mit fehlenden Werten umgegangen werden soll. Zwei Extremfälle sind denkbar:

1) Es werden alle Beobachtungen ausgeschlossen, in denen eine der Teilfragen nicht beantwortet wurde. In diesem Fall fließen in die Schätzung nur diejenigen Befragten ein, die alle Teilfragen beantwortet haben. Bei diesem Vorgehen verbleiben nur 59 gültige Beobachtungen in der PL- und 26 in der PM-Stichprobe.

2) Es werden für die Mittelwertbildung jeweils nur diejenigen Teilfragen berücksichtigt, welche beantwortet wurden. Fehlende Werte entstehen hier nur dann, wenn ein Teilnehmer keine der 14 Teilfragen beantwortet hat. Dieser Fall tritt in der PL-Stichprobe viermal und in der PM-Stichprobe einmal auf, so dass 133 bzw. 43 Beobachtungen verbleiben.

Des Weiteren sind auch Mischformen denkbar. Beispielsweise können solche Beobachtungen ausgeschlossen werden, in denen mehr als die Hälfte der Teilfragen von Frage 28 nicht beantwortet wurden. Bei dieser Option verlieren wir in der PL-Stichprobe 15 Beobachtungen, in der PM-Stichprobe nur eine Beobachtung. Wir halten diese Vorgehensweise für einen geeigneten Kompromiss und verwenden sie für die hier ausführlich präsentierten Regressionen. Abgesehen davon, kommen auch andere Aggregationsmög-

41 Alle Datenanalysen und Schätzungen wurden mit den Programmen Stata 8 oder SPSS 12 durchgeführt.

42 Darüber hinaus konnte die Antwort „weiß nicht“ gegeben werden. Beobachtungen mit dieser Antwort wurden in den Regressionen mit Frage 28 als abhängiger Variable jedoch ausgeschlossen.

43 Die Anteile beziehen sich auf alle gültigen Antworten, d.h. ohne fehlende Werte oder solche mit der Antwort „weiß nicht“. In der PL-Stichprobe werden somit 5,1\% der Beobachtungen ausgeschlossen, in jener der Projektmitarbeiter sind dies $4,5 \%$. 
lichkeiten der Variablen $f 28$ in Frage, z.B. Faktorwerte, welche wir in Vergleichsschätzungen (vgl. Abschnitt 6.2.3) berücksichtigen.

In allen Teilfragen von Frage 28 indizieren kleinere Werte eine höhere Projektzufriedenheit auf einer Skala von 1 (= trifft vollständig zu) bis 4 (= trifft gar nicht zu). Der Mittelwert der Antworten in der PL-Stichprobe beträgt 2,26 und jener der PM-Stichprobe 2,4344. Damit zeigt sich auch in Bezug auf die Projekt-Teilziele eine etwas höhere Projektzufriedenheit der Projektleiter.

Die Antworten zu den Fragen 27 und 28 zeigen somit, dass die Projektmitarbeiter die Projekterfolge generell weniger positiv beurteilen als die Projektleiter. Der Unterschied ist gemäß einem $t$-Test auf dem 10\%-Niveau statistisch signifikant ${ }^{45}$. Unsere Ergebnisse liefern somit zumindest einen Anhaltspunkt für eine unterschiedliche Projektbewertung durch Betroffene und Führungskräfte. Eine nahe liegende Interpretation wäre, dass der „Blick von unten“ weniger positiv ist, als der „Blick von oben“.

Da die Untersuchung relativ breit angelegt ist, enthält die Datenbasis eine Vielzahl von (potenziell) erklärenden Variablen. Für die Fragestellung der vorliegenden Arbeit wurden diese anhand theoretischer Überlegungen auf 14 besonders relevante Variablen reduziert (siehe Abbildung 1). Anschließend wurde eine weitere Datenreduktion anhand der $t$-Statistiken vorgenommen, das heißt, es wurden nur Kombinationen von Variablen verwendet, von denen jede einzelne in den OLS-Ausgangsschätzungen ${ }^{46}$ mit der PL-Stichprobe auf dem 5\%-Niveau signifikant war. Alle von uns verwendeten erklärenden Variablen wurden auf einer Skala von 1 bis 4 bewertet, diese sind in Tabelle 2 zusammengefasst. Alle berücksichtigten Variablen wurden in beiden Stichproben (PL und PM) erhoben, mit Ausnahme von Variable $f 12 \mathrm{a}$ (klare Zielvorgaben), welche nur in der Befragung der Projektleiter auftrat und in Regressionsschätzungen mit der PM Stichprobe durch die Variable $f 12 \mathrm{~b}$ (Detaillierungsgrad der Zielvorgaben) ersetzt wurde.

Die erklärenden Variablen sind untereinander paarweise nicht sehr hoch korreliert (der maximale Korrelationskoeffizient, jener zwischen $f 10$ und $f 25$, beträgt in der PL-Stichprobe knapp 0,6, der zweithöchste beträgt 0,48). Dies ist nicht selbstverständlich, da ein erster Blick auf die Verteilung der Werte in Tabelle 2 beispielsweise suggeriert, dass Fragen $12 \mathrm{a}$ und 15 in der PL Stichprobe von den gleichen Personen gleich beantwortet wurden. Tatsächlich beträgt der Korrelationskoeffizient zwischen beiden Variablen aber weniger als 0,2. Betrachtet man Kollinearitäten zwischen mehreren Variablen, so ergibt sich in linearen Regressionen der erklärenden Variablen aufeinander mit der PL-Stichprobe ein maximales $R^{2}$ von 0,46 ; dies entspricht einem Varianzinflationskoeffizienten $(V I F)$ von 1,85 .

44 Die Standardabweichungen sind 0,56 bzw. 0,61.

45 Sowohl für die Variable $f 27$ als auch für die Variable $f 28$ wird die Hypothese, dass ihr Mittelwert in beiden Stichproben gleich ist, auf dem 10\% Niveau gemäß dem $t$-Test (Annahme kardinale Skalierung und gleiche Varianzen, welche gemäß Levene-Test angenommen werden können) knapp verworfen, auf der Basis von nichtparametrischen Mann-Whitney U-Tests jedoch knapp gestützt.

46 OLS steht für „Ordinary Least Squares for Multiple Regression“. Ausgangsschätzungen sind OLS Schätzungen mit der abhängigen Variable $f 28$ (Modelle 1 und 2 in Tabelle 3). 
Tabelle 2: Beschreibung der verwendeten erklärenden Variablen

\begin{tabular}{|c|c|c|c|c|c|}
\hline \multirow[t]{2}{*}{ Nr. } & \multirow[t]{2}{*}{ Frage/Aussage } & \multicolumn{2}{|c|}{$\begin{array}{l}\text { Mögliche Antworten } \\
\text { (Mediane fett gedruckt) }\end{array}$} & \multicolumn{2}{|c|}{$\begin{array}{l}\text { Verteilung der Antworten absolut } \\
\text { ( } \% \text { an gültigen Werten) }\end{array}$} \\
\hline & & & & PL & PM \\
\hline$f 10$ & $\begin{array}{l}\text { Die wichtigsten vom } \\
\text { Projekt betroffenen } \\
\text { Gruppen haben im } \\
\text { Projekt tatsächlich } \\
\text { mitgewirkt. }\end{array}$ & $\begin{array}{l}1 \\
2 \\
3 \\
4\end{array}$ & $\begin{array}{l}\text { Trifft vollständig zu } \\
\text { Trifft eher zu } \\
\text { Trifft eher nicht zu } \\
\text { Trifft gar nicht zu }\end{array}$ & $\begin{array}{r}35(26,32) \\
69(51,88) \\
26(19,55) \\
\quad 3(2,26)\end{array}$ & $\begin{array}{r}10(22,73) \\
24(54,55) \\
9(20,45) \\
1(2,27)\end{array}$ \\
\hline$f 12 a$ & $\begin{array}{l}\text { Wie waren die Zielvor- } \\
\text { gaben des Projekts? }\end{array}$ & $\begin{array}{l}1 \\
2 \\
3 \\
4\end{array}$ & $\begin{array}{l}\text { Sehr klar } \\
\text { Klar } \\
\text { Eher unklar } \\
\text { Sehr unklar }\end{array}$ & $\begin{array}{r}14(10,53) \\
92(63,16) \\
24(24,81) \\
\quad 4(1,50)\end{array}$ & \\
\hline$f 12 b$ & $\begin{array}{l}\text { Wie waren die Zielvor- } \\
\text { gaben des Projekts? }\end{array}$ & $\begin{array}{l}1 \\
2 \\
3 \\
4\end{array}$ & $\begin{array}{l}\text { Sehr detailliert } \\
\text { Eher detailliert } \\
\text { Eher grob } \\
\text { Sehr grob }\end{array}$ & & $\begin{array}{r}3(6,98) \\
26(60,47) \\
9(20,93) \\
5(11,63)\end{array}$ \\
\hline$f 15$ & $\begin{array}{l}\text { Wie war das Engage- } \\
\text { ment der wichtigsten } \\
\text { Entscheidungsträger? }\end{array}$ & $\begin{array}{l}1 \\
2 \\
3 \\
4\end{array}$ & $\begin{array}{l}\text { Vorbehaltlos } \\
\text { Deutlich } \\
\text { Eher schwach } \\
\text { Kaum vorhanden }\end{array}$ & $\begin{array}{r}13(9,77) \\
92(69,17) \\
24(18,05) \\
4(3,01)\end{array}$ & $\begin{array}{r}5(11,36) \\
22(50,00) \\
15(34,09) \\
2(4,55)\end{array}$ \\
\hline$f 16$ & $\begin{array}{l}\text { Wie beurteilen Sie } \\
\text { die Entscheidungen } \\
\text { der Steuerungsverant- } \\
\text { wortlichen? }\end{array}$ & $\begin{array}{l}1 \\
2 \\
3 \\
4\end{array}$ & $\begin{array}{l}\text { Sehr kompetent } \\
\text { Kompetent } \\
\text { Nicht besonders } \\
\text { kompetent } \\
\text { Inkompetent }\end{array}$ & $\begin{array}{r}4(3,03) \\
98(74,24) \\
29(21,97) \\
1(0,76)\end{array}$ & $\begin{array}{r}2(4,65) \\
27(62,79) \\
12(27,91) \\
2(4,65)\end{array}$ \\
\hline$f 17$ & $\begin{array}{l}\text { Entscheidungen des } \\
\text { Steuerungskomitees } \\
\text { waren sachlich oder } \\
\text { interessenbedingt. }\end{array}$ & $\begin{array}{l}1 \\
2 \\
3 \\
4\end{array}$ & $\begin{array}{l}\text { Überwiegend sachlich } \\
\text { Meist sachlich } \\
\text { Meist interessenbedingt } \\
\text { Überwiegend interessen- } \\
\text { bedingt }\end{array}$ & $\begin{array}{l}15(11,28) \\
69(51,88) \\
35(26,32) \\
14(10,53)\end{array}$ & $\begin{array}{r}3(6,98) \\
21(48,84) \\
9(20,93) \\
10(23,26)\end{array}$ \\
\hline$f 25$ & $\begin{array}{l}\text { Die Projektdurchführung } \\
\text { war von einem ganzheit- } \\
\text { lichen Ansatz geprägt. }\end{array}$ & $\begin{array}{l}1 \\
2 \\
3 \\
4\end{array}$ & $\begin{array}{l}\text { Trifft vollständig zu } \\
\text { Trifft eher zu } \\
\text { Trifft eher nicht zu } \\
\text { Trifft gar nicht zu }\end{array}$ & $\begin{array}{r}17(12,98) \\
76(58,02) \\
31(23,66) \\
7(5,34)\end{array}$ & $\begin{array}{r}7(17,50) \\
23(57,50) \\
7(17,50) \\
3(7,50)\end{array}$ \\
\hline
\end{tabular}

47 Die Mediane unterscheiden sich zwischen der PL und PM Stichprobe nicht. 
In Regressionen mit der PM-Stichprobe beträgt das entsprechende $R^{2} 0,49$ (bzw. VIF = $1,98)$. Damit treten in unseren nachfolgend präsentierten Regressionsschätzungen keine bedeutsamen Multikollinearitätsprobleme auf (solche werden je nach Autor bei VIFWerten größer als 10 oder 30 konstatiert ${ }^{48}$.

Die Mediane aller erklärenden Variablen (wie auch der abhängigen Variablen) betragen in beiden Stichproben 2; dies zeigt in der Regel eine Zustimmung oder positive Bewertung der Frage/Aussage an.

Das Ergebnis der Datenreduktion auf Basis der $t$-Werte zeigt, dass unabhängige Variablen aus allen drei Kategorien selektiert wurden (vgl. Abbildung 1):

- Aktivitäten: $f 12$ (Zielvorgaben), $f 25$ (ganzheitlicher Ansatz)

- Strukturen: $f 10$ (partizipatives Management)

- Verhalten: $f 15$ (Engagement der Entscheidungsträger), $f 17$ (Fokus der Entscheidungen), $f 16$ (Kompetenz der Entscheidungsträger)

Dies stützt die oben genannte Arbeitshypothese, dass bei Untersuchungen über das Projektmanagement die verschiedenen Aspekte integrativ berücksichtigt werden sollten.

Zweitens überrascht bei den durch die Datenreduktion eliminierten Variablen besonders das Wegfallen von „Autonomie der Projekte“ und „Spielräume für Projektmitarbeiter“. Diese zählen von allen herausgefilterten Größen zu den am breitesten gefassten. Zudem werden sie heute als Grundprinzipien effektiven Organisationsdesigns betrachtet ${ }^{49}$, was für die anderen weggefallenen Variablen nicht gilt.

\subsection{Regressionsschätzungen}

\subsubsection{Vorüberlegungen}

Ausführlich diskutiert werden Schätzungen für Modelle mit der Projektleiter (PL-) Stichprobe und $f 28$ (Beurteilung der Ergebnisse und Wirkungen) als abhängiger Variable. Diese Wahl ist darin begründet, dass erstens die PL-Stichprobe wesentlich mehr Beobachtungen als die PM-Stichprobe enthält, und zweitens die Variable $f 28$ als Indikatorvariable mit 14 Teilfragen und 4 Kategorien verschiedene Facetten der Projektevaluation abdeckt. Ergänzend werden aber auch Ergebnisse von Schätzungen der PM-Stichprobe und solchen mit der Variable $f 27$ (ex post Meinung über das Projekt) angesprochen (vgl. Abschnitt 6.2.3).

Für die Wahl des geeigneten Regressionsmodells ist die Frage zu entscheiden, welche Art der Skalierung (kardinal, ordinal oder nominal) angenommen werden kann. Alle unsere Variablen sind in jedem Fall zumindest ordinalskaliert, da sie eine Rangfolge der Beur-

49 Vgl. Goold/Campbell (2002); Rüegg-Stürm/Young (2001). 
teilung beinhalten. Wir nehmen darüber hinaus an, dass sie kardinalskaliert sind, d.h. wir gehen von gleichen Abständen zwischen benachbarten Beurteilungswerten aus. Diese Annahme ist bei Studien mit vergleichbaren Befragungsdaten allgemein üblich, unter anderem, weil es hierdurch möglich ist, relativ leicht interpretierbare OLS-Schätzungen vorzunehmen ${ }^{50}$. $\mathrm{Zu}$ Vergleichszwecken haben wir jedoch auch ordinale Regressionsmodelle geschätzt, in denen sowohl die Variablen der rechten als auch jene der linken Seite als ordinalskaliert angenommen werden (siehe Abschnitt 6.2.3).

Schließlich ist noch ein weiterer Punkt zu berücksichtigen. Wie oben bereits erwähnt, stammen die Mitarbeiter aus neun unterschiedlichen Unternehmen (bzw. Branchen) ${ }^{51}$. Es ist in Frage zu stellen, dass die einzelnen Beobachtungen innerhalb der Unternehmen tatsächlich unabhängig sind; dies bedeutet, dass eine Annahmeverletzung des OLSModells vorliegt. Das Problem kann durch die Verwendung der Stata-Option „Cluster“ berücksichtigt werden ${ }^{52}$. In unserem Fall werden die einzelnen Unternehmen (bzw. Branchen) als Cluster gewählt. Notwendige Voraussetzung dieser Modelle ist nunmehr nur, dass die Beobachtungen zwischen den Unternehmen unabhängig sind. Gegenüber Schätzungen ohne diese Option ändern sich die Koeffizienten nicht, jedoch erhält man andere Standardfehler und damit andere $t$-Werte.

\subsubsection{Schätzergebnisse}

A priori war nicht klar, welche der aus theoretischen Vorüberlegungen in Frage kommenden Variablen tatsächlich einen substanziellen Einfluss auf die Gesamtbeurteilung des Projekts ausüben. Verschiedene Spezifikationen wurden getestet, und es wurden nur Modelle berücksichtigt, bei denen alle erklärenden Variablen in der OLS-Regression mit Clustering signifikant waren ${ }^{53}$. Hierbei wurden zwei alternative Spezifikationen mit drei bzw. fünf erklärenden Variablen gefunden (Modelle 1 und 2 in Tabelle 3). Darüber hinaus wurde noch ein Modell geschätzt, welches alle in den beiden vorherigen Regressionen verwendeten Variablen enthält (Modell 3 in Tabelle 3). Die Variable $f 17$ (Fokus der Entscheidungen) hat hier keinen signifikanten Einfluss mehr ${ }^{54}$. Ein Vergleich der Modelle zeigt unabhängig davon aber, dass die Vorzeichen der Koeffizienten und damit die Richtungen des Einflusses der erklärenden Variablen auf die abhängige Variable (Projektzufriedenheit ex post) immer gleich sind. Dies unterstreicht die Zuverlässigkeit der Ergebnisse der einzelnen Teilmodelle auch nach der Kontrolle für andere potenziell relevante Einflussgrößen.

Einzelne Studien zeigen jedoch auch, dass (z.B. OLS-) Schätzungen unter einer fälschlichen Annahme von Kardinalskalierung irreführende Ergebnisse liefern können (Winship/Mare 1984); außerdem können Probleme wie Heteroskedastizität auftreten (vgl. Long (1997), S. 118).

51 Jedes Unternehmen gehört einer anderen Branche an.

52 Vgl. Rogers (1993).

53 Basis der Auswahl war ein 10\% Signifikanzniveau. Wie sich zeigt, wird aber auf den 5\% und 1\% Niveaus dieselbe Auswahl getroffen.

54 Dies hängt mit Korrelationen zwischen den erklärenden Variablen zusammen. 
Tabelle 3: Schätzergebnisse von OLS-Schätzungen mit f28 (Ergebnisse und Wirkungen des Projekts) als abhängiger Variable für die PL-Stichprobe

\begin{tabular}{lccc}
\hline & Modell 1 & Modell 2 & Modell 3 \\
\hline$f 10$ & $0,235^{* * *}$ & $0,118^{* * *}$ & $0,114^{* *}$ \\
(Partiz. Management) & $(0,014)$ & $(0,033)$ & $(0,035)$ \\
$f 17$ & $0,196^{* * *}$ & & 0,068 \\
(Fokus d. Entscheidungen) & $(0,051)$ & & $(0,050)$ \\
$f 15$ & $0,337^{* * *}$ & $0,290^{* * *}$ & $0,291^{* * *}$ \\
(Engagement d. Entsch.träger) & $(0,047)$ & $(0,033)$ & $(0,037)$ \\
$f 12 a$ & & $0,166^{* * *}$ & $0,175^{* * *}$ \\
(klare Zielvorgaben) & & $(0,047)$ & $(0,042)$ \\
$f 16$ & & $0,366^{* * *}$ & $0,277^{* * *}$ \\
(Kompetenz d. Verantwortlichen) & & $(0,064)$ & $(0,074)$ \\
$f 25$ & & $0,114^{* * *}$ & $0,099^{* * *}$ \\
(Ganzheitlicher Ansatz) & & $(0,023)$ & $(0,025)$ \\
Konstante & $0,639^{* * *}$ & 0,032 & 0,081 \\
& $(0,128)$ & $(0,158)$ & $(0,129)$ \\
\hline$R^{2}$ & 0,480 & 0,550 & 0,558 \\
Korrigiertes $R^{2}$ & 0,466 & 0,527 & 0,531 \\
$F$-Statistik & 306,002 & 171,966 & 117,431 \\
Pr (F) & 0,000 & 0,000 & 0,000 \\
$N$ & 113,000 & 107,000 & 105,000 \\
\hline${ }^{*} p<0,1, * * p<0,05, * * * p<0,01$ & & & \\
Robuste Standardfehler in Klammern, & & & \\
Cluster-Variable: Unternehmen & & & \\
\hline
\end{tabular}

Aus den Koeffizienten lässt sich ersehen, dass die Projekte ex post um so besser bewertet wurden,

- je höher das Engagement der wichtigsten Entscheidungsträger $(f 15)$,

- je kompetenter die Entscheidungen der Steuerungsverantwortlichen $(f 16)$,

- je ganzheitlicher der Ansatz der Projektdurchführung ( $f 25)$,

- je mehr die vom Projekt betroffenen Gruppen tatsächlich mitwirkten $(f 10)$,

- je stärker die Entscheidungen des Steuerungskomitees sachlich bedingt waren $(f 17)$,

- je klarer die Zielvorgaben des Projektes waren $(f 12 a)$. 


\subsubsection{Vergleichsschätzungen}

Die Ergebnisse der oben präsentierten Schätzung wurden mit den Ergebnissen weiterer Schätzungen verglichen. Deren Resultate geben wir hier nicht detailliert an, sie können aber bei Interesse von den Autoren zur Verfügung gestellt werden.

\section{1) Schätzungen mit der PM-Stichprobe}

Schätzungen mit der Stichprobe von Projektmitarbeitern ergeben ähnliche Ergebnisse im Hinblick auf die qualitativen Aussagen wie die im letzten Abschnitt präsentierten. In Anbetracht der deutlich kleineren Stichprobengröße ist die Zuverlässigkeit der Parameterschätzung allerdings geringer. Ein Vergleich der Koeffizienten zeigt, dass die marginalen Einflüsse der erklärenden Variablen auf die Projektzufriedenheit bei den Projektmitarbeitern generell kleiner sind als bei den Projektleitern. Nur bei Variable $f 15$ (Engagement der Entscheidungsträger) ist dies umgekehrt, d.h. die Bedeutung des Engagements der Entscheidungsträger für den Projekterfolg wird von den Projektmitarbeitern höher eingeschätzt als von den Projektleitern.

\section{2) Alternative Berechnungen der Indikatorvariable $f 28$}

Wie bereits erwähnt, bestehen verschiedene Möglichkeiten, die Variable $f 28$ aus ihren 14 Teilfragen zu generieren. In der oben präsentierten Schätzung haben wir nur solche Beobachtungen verwendet, in denen mindestens die Hälfte der Teilfragen beantwortet wurde. In einer alternativen Reihe von Schätzungen wurden stattdessen alle Befragten berücksichtigt, die mindestens eine der Teilfragen beantwortet haben. Die Ergebnisse dieser Schätzungen waren sehr ähnlich (alle Vorzeichen und Signifikanzniveaus der Koeffizienten bleiben unverändert). Im Wesentlichen können wir eine leichte Verschlechterung des $R^{2}$ beobachten (in Modell 3 auf einen Wert von 0,528).

Eine weitere Möglichkeit für die Aggregation der einzelnen Teilfragen besteht darin, als abhängige Variable den Faktorwert (factor score) des ersten Faktors zu verwenden ${ }^{55}$. Bei der Berechnung der Faktorwerte wiederholt sich das oben bereits diskutierte Problem, dass es in der Projektleiterstichprobe nur 59 Beobachtungen gibt, die keine fehlenden Werte für mindestens eine der Teilfragen von Frage 28 aufweisen. Schätzungen mit Faktorwerten auf Basis dieser 59 Beobachtungen führen zu keinen Änderungen hinsichtlich der Vorzeichen der Koeffizienten im Vergleich zu den bereits angesprochenen Schätzergebnissen; aber wir haben bei erwartungsgemäß geringeren Signifikanzniveaus der Koeffizienten gleichzeitig höhere Bestimmtheitsmasse (z.B. erhalten wir in Modell 3 ein $R^{2}$ von 0,654 und ein korrigiertes $R^{2}$ von 0,612 ).

55 Vgl. Hair et al. (1998). Das hier angesprochene Vorgehen beinhaltet, dass eine Faktorenanalyse durchgeführt wird. Diese führt zur Identifikation latenter Faktoren, welche verschiedene Dimensionen der Projektzufriedenheit beschreiben. Der erste Faktor ist derjenige Faktor, welcher den größten Teil der Varianz aller Teilfragen von $f 28$ erfasst („Hauptzufriedenheitsfaktor“). Der Faktorwert einer Person beschreibt, wie stark dieser Hauptzufriedenheitsfaktor in einer Person ausgeprägt ist. 


\section{3) Schätzungen mit der endogenen Variable $f 27$ sowie ordinale Regressionen}

Wie erwähnt, kann statt der Variable $f 28$ auch die Variable $f 27$ (Meinung über das Projekt) verwendet werden, welche drei Ausprägungen annimmt. Wieder erhält man ähnliche Aussagen wie in unserem Ausgangsmodell bei einem etwas schlechteren $R^{256}$.

Interessanter ist ein Vergleich mit einer weiteren Reihe von Schätzungen mit der abhängigen Variable $f 27$, in der alle Variablen als ordinalskaliert angenommen werden. Geschätzt wurden ordinale logistische Regressionsmodelle mit dummy-codierten erklärenden Variablen. Ordinale logistische Regressionsmodelle sind geeignet, wenn die abhängige Variable ordinalskaliert ist, d.h. die Kategorien weisen eine Rangfolge auf, jedoch ist die Distanz zwischen benachbarten Kategorien unbekannt. Zu Details dieser Modelle siehe Long (1998) ${ }^{57}$. Im Fall der Projektbeurteilung ist es denkbar, dass sich die Abstände zwischen den einzelnen Antwortkategorien unterscheiden. Falls sich die mit diesem Modell generierten Aussagen grundsätzlich von jenen aus dem linearen Regressionsmodell unterscheiden, ist die Annahme kardinalskalierter Variablen in Frage zu stellen.

Die Schätzergebnisse des ordinalen Modells mit Variable $f 27$ als abhängiger Variable zeigen, dass die Annahme der Gleichabständigkeit in Bezug auf die erklärenden Variablen zwar generell problematisch ist, es fällt jedoch auch auf, dass die Einflüsse der einzelnen Variablen streng monoton in dem Sinn sind, dass ein Anstieg der erklärenden (ordinalen) Variablen generell mit einem Anstieg der abhängigen Variablen einhergeht. Dies bedeutet kurz gesagt: Die Eigenschaft konstanter marginaler Effekte, wie sie beim linearen Regressionsmodell vorausgesetzt wird, ist nicht zutreffend. Die Richtung des Einflusses der Regressoren auf die abhängige Variable wird aber zuverlässig sowohl aus den linearen wie auch aus den ordinalen Modellen ersichtlich.

Aus den Schätzergebnissen des ordinalen Regressionsmodells mit dummy-codierten Regressoren lassen sich darüber hinaus zusätzliche, praxisrelevante Implikationen für die einzelnen Variablen ableiten.

In Abbildung 2 ist die Wahrscheinlichkeit für eine gute $(f 27=1)$, mittlere $(f 27=2)$ und schlechte $(f 27=3)$ Projektbeurteilung dargestellt, in Abhängigkeit von der Variablen $f 15$ (Engagement der Entscheidungsträger), wie sie aus der ordinalen logistischen Regression resultiert. Alle anderen Variablen werden auf ihre Mittelwerte fixiert. Es wird deutlich, dass ein Wechsel der Variablen $f 15$ vom Wert 1 (vorbehaltlos) auf den Wert 2 (deutlich) eine wesentlich stärkere Auswirkung auf die Projektzufriedenheit hat als ein Wechsel vom Wert 3 (eher schwach) auf den Wert 4 (kaum vorhanden). Ähnliches gilt für die Variablen $f 10, f 12$ und $f 17$. Das bedeutet, dass es für diese Variablen wirksamer ist, das Engagement dort zu fördern, wo das Ausgangsniveau bereits hoch ist, als dort, wo es nicht vorhanden oder nur niedrig ist. Dieses Ergebnis stützt das Prinzip, auf vorhandenen Stärken aufzubauen ${ }^{58}$.

56 In Modell 3 wird beispielsweise nur noch ein Wert von 0,340 erreicht. Zudem sind nun (auch) in Modell 2 nicht mehr alle Koeffizienten signifikant.

57 Bei der Dummy-Codierung wird für jeden Wert einer nominalen Variable eine binäre Variable geschaffen, welche den Wert 1 annimmt, wenn die entsprechende nominale Variable diesen Wert aufweist.

58 Vgl. Drucker (1993); Pümpin/Geilinger (1988). 


\section{Abbildung 2: Wahrscheinlichkeit einer guten, mittleren und schlechten Projektbeurteilung für verschiedene Werte von $\boldsymbol{f}^{15} 5^{59}$}

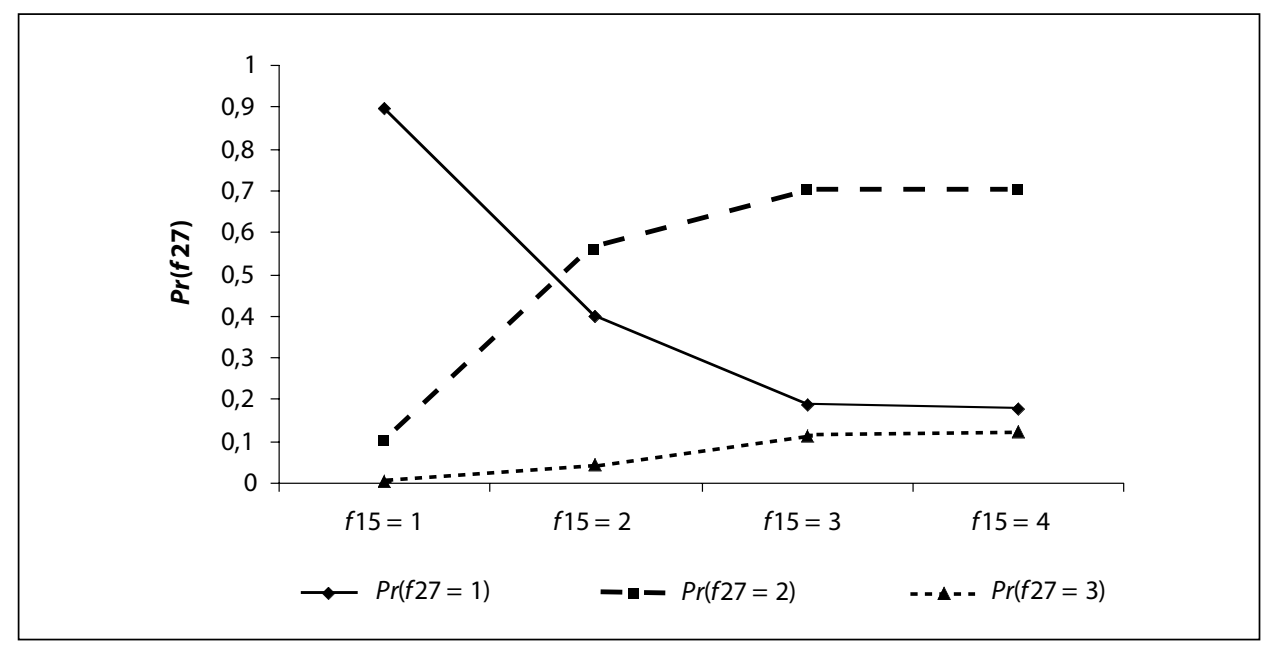

\section{Fazit}

In diesem Beitrag sind wir der Frage nach den Erfolgsfaktoren von Organisations- und Veränderungsprojekten nachgegangen. Zu diesem Zweck wurde eine bei Projektleitern und Projektmitarbeitern durchgeführte Befragung mit verschiedenen Modellen statistisch ausgewertet. Die Ergebnisse der Modellschätzungen erwiesen sich als robust gegenüber verschiedenen Modellannahmen und zeigen den deutlichen Einfluss bestimmter kritischer Faktoren auf den Projekterfolg.

Inhaltlich gesehen führten die Analysen zu einer Reihe von Ergebnissen, die hier zusammengefasst werden. Dies mit Blick auf die eingangs gestellten Forschungsfragen, kurz: Welche sind die Erfolgsfaktoren von Organisations- und Veränderungsprojekten und wirken sie auf verschiedenartige Erfolgsgrößen unterschiedlich? Jeder einzelnen Erkenntnis werden wir Implikationen für Projektleiter und andere in Gremien der Projektsteuerung tätige Führungskräfte gegenüberstellen.

1.) Eine Reihe von Faktoren zeigt einen signifikanten Einfluss auf den Erfolg von Organisations- und Veränderungsprojekten:

- klare Zielvorgaben,

- das Engagement der Entscheidungsträger,

59 Die Grafik bezieht sich auf eine ordinale logistische Regression mit der abhängigen Variable $f 27$ und der PLStichprobe. 
- die Kompetenz der Steuerungsverantwortlichen,

- ein ganzheitlicher Ansatz des Projektmanagements,

- die Mitwirkung der vom Projekt betroffenen Gruppen,

- eine sachliche, nicht interessenbezogene Orientierung der Entscheidungen.

Die Zusammenhänge zwischen diesen erklärenden Variablen und den Erfolgsgrößen sind in dieser Studie untermauert worden. Sie widersprechen der im 4. Abschnitt zitierten Basisliteratur nicht. Es sind uns zwar keine Untersuchungen bekannt, die mit dieser, spezifisch auf Organisations- und Veränderungsprojekte zugeschnittenen Studie direkt vergleichbar wären. Allerdings zeigen sich markante Parallelen zu Forschungsergebnissen bezüglich der Erfolgsfaktoren von Projekten im Allgemeinen. Eine Meta-Analyse der Ergebnisse von 5.700 Projekten identifiziert immerhin vier stark erfolgswirksame Faktoren, die sich mit den oben genannten weitestgehend decken: Partizipation der Projektteammitglieder, klare und verbindliche Zieldefinition, Engagement von Machtpromotoren, Führungsverhalten der Projektleiter ${ }^{60}$. Die beiden anderen hier als signifikant identifizierten Einflussgrößen - ganzheitlicher Ansatz und sachliche Orientierung - figurieren dort nicht explizit.

Implikation: Es mag viele Faktoren geben, die in irgendeiner Form erfolgswirksam sind. Die vorliegenden Ergebnisse sprechen jedoch dafür, dass der Projekterfolg bei Organisations- und Veränderungsprojekten vor allem von den hier herausgearbeiteten Faktoren abhängt, welche im Führungsprozess besonders beachtet werden sollten.

2.) Projektleiter und Projektmitarbeiter gelangen bezüglich der unter 1.) dargelegten Zusammenhänge praktisch zu denselben Beurteilungen. Dies gilt mit einer Ausnahme: Die Mitarbeiter haben generell eine weniger positive Sicht auf die Ergebnisse der Projekte als die Mitarbeiter. Dieses Ergebnis wurde durch uns weder antizipiert noch in der einschlägigen Literatur anderweitig bestätigt.

Implikation: Es ist wichtig, Mitarbeiter in systematische Evaluationsprozesse (Monitoring, Manöverkritik usw.) einzubinden. Auf diese Weise kommt nicht nur eine gemeinsame Beurteilung des Projekterfolges zustande. Vielmehr ergeben sich daraus hochwertige Möglichkeiten für organisationales Lernen. Dies speziell auf Teamebene ${ }^{61}$ und auch im Sinne eines Lernens höherer Ordnung ${ }^{62}$.

3.) Die Analyseergebnisse bezüglich der Feinstrukturen bei den Zusammenhängen zwischen den Wirkfaktoren und den Erfolgsgrößen haben ein bewährtes Führungsprinzip bestätigt: das Primat des Aufbaus auf Stärken.

Dieses Ergebnis wurde in der Anlage der Untersuchung nicht speziell antizipiert. Es ist zwar von einigen Autoren hervorgehoben worden, aber systematische empirische Unter- 
suchungen in Bezug auf dieses Prinzip fehlen soweit uns bekannt ist. Vor allem aber zeigt die Praxis große, weithin sichtbare Umsetzungsdefizite ${ }^{63}$.

Implikation: Die Führungskräfte sollten sich mit Nachdruck an das Prinzip des Verstärkens von Stärken erinnern, dem gegenüber einem Beseitigen von Schwächen klare Priorität einzuräumen ist.

4.) Die eruierten Zusammenhänge zwischen Erfolgsfaktoren und Erfolgsmaßstäben sind robust. Sie gelten gleichermaßen für unterschiedliche Erfolgsgrößen, konkret für „Zufriedenheit mit Projektergebnissen“, mehr noch für "Ergebnisse und Wirkungen des Projekts“. Dabei ist zu differenzieren; nicht alle Erfolgsfaktoren haben eine gleich starke Wirkung auf die abhängigen Variablen. Am stärksten wirken „Engagement der Entscheidungsträger“, „sachlicher Fokus der Entscheidungen“ und „klare Zielsetzungen“64.

Implikation: Führungskräfte können davon ausgehen, dass die hier herausgearbeiteten Erfolgsfaktoren mit relativ hoher Zuverlässigkeit auf unterschiedliche Erfolgsgrößen wirken. Zudem sollten sie die soeben hervorgehobenen Größen als „Hebel“ mit besonders hoher Steuerungswirksamkeit verstehen. Sie weisen nämlich den stärksten Hebeleffekt („leverage“) auf.

Abschließend ist auf die spezifischen Grenzen dieser Aussagen hinzuweisen. Erstens reflektieren dieselben keine gesetzesartigen Zusammenhänge. Sie sind vielmehr Tendenzaussagen. Im Einzelfall können die Zusammenhänge von der jeweils dargestellten Tendenz abweichen. Zweitens besteht keine Garantie, dass die Studie alle prinzipiell möglichen Erfolgsfaktoren lückenlos erfasst. Drittens ist die Stichprobe auf acht Unternehmen beschränkt, bildet also nicht die gesamte Population von Unternehmungen ab. Trotz dieser Einschränkungen stellen die Ergebnisse zumindest einen relevanten Anfang dar. Einer explorativen Studie gemäß können sie eine indikative Funktion erfüllen.

Als Fortsetzung dieser Untersuchung wäre eine Wiederholung bei einer größeren Stichprobe sinnvoll. Zudem wäre die Durchführung einer speziell angepassten Befragung mit einer Stichprobe von Klein- und Mittelbetrieben von Interesse. Darüber hinaus sollten in zukünftigen Studien unterschiedliche Organisationsformen und Vorgehensweisen bei Veränderungsprojekten auf ihre Erfolgswirksamkeit geprüft werden. Zudem sollte geprüft werden, inwiefern unterschiedliche Verhaltenstypen, z.B. die von Freudenberg (1999) eruierten, zu Variationen bei der Erfolgserzielung führen. Schließlich sollten auch die Erfolgsfaktoren für andere Projekttypen in ähnlicher Weise untersucht werden.

64 Vgl. Lechler (2005). Die genannte Priorisierung tritt erst in den hier vorgelegten Ergebnissen zutage. Aufgrund der Basisliteratur waren die genannten drei Größen zwar als Kandidaten für Erfolgsfaktoren identifizierbar, aber nicht hinsichtlich ihres Gewichtes einschätzbar. 


\section{Literatur}

Adner, Ron/Helfat, Constance E. (2003), Corporate Effects and Dynamic Managerial Capabilities, in: Strategic Management Journal, Vol. 24, S. 1011-1025.

Andress, Hans-Jürgen/Hagenaars, Jacques A./Kühnel, Steffen (1997), Analyse von Tabellen und kategorialen Daten: Log-lineare Modelle, latente Klassenanalyse, logistische Regression und GSK Ansatz, Berlin.

Argyris, Chris (1999), On Organizational Learning, 2. Aufl., Cambridge, Massachusetts/Oxford.

Bauer, Hans H./Sauer, Nicola E. (2004), Die Erfolgsfaktorenforschung als schwarzes Loch?, in: Die Betriebswirtschaft, 64. Jg., S. 621-623.

Beer, Stafford (1979), Heart of Enterprise, Chichester et al.

Bleicher, Knut (2004), Das Konzept. Integriertes Management: Visionen - Missionen - Programme, 7. Aufl., Frankfurt/New York.

Bruch, Heike (2003), Leaders' Action, München.

Chatterjee, Samprit/Hadi, A.S./Price, B. (2000), Regression Analysis by Example, 3. Aufl., New York.

Cremer, Jörg (2002), Motivation in Projekten. Eine empirische Erforschung von Motivations- und Erfolgsursachen im Projektmanagement, Dissertation, Universität Köln, Lengerich et al.

Deci, Edward L./Ryan, Richard M. (1993), Die Selbstbestimmungstheorie der Motivation und ihre Bedeutung für die Pädagogik, in: Zeitschrift für Pädagogik, 39. Jg., S. 223-237.

Dess, Gregory G./Robinson, Richard B. (1984), Measuring Organizational Performance in the Absence of Objective Measures: The Case of the Privately-held Firm and Conglomerate Business Unit, in: Strategic Management Journal, Vol. 5, S. 265-273.

Diekmann, Andreas (1998), Empirische Sozialforschung, 4. Aufl., Reinbek.

Drucker, Peter F (1974), Neue Management-Praxis, 2. Band, Düsseldorf/Wien.

Drucker, Peter F. (1993), Managing for Results, reprint edition, New York (ursprünglich veröffentlicht 1964).

Dvir, Dov et al. (1998), In Search of Project Classification: a Non-universal Approach to Project Success Factors, in: Research Policy, Vol. 27, S. 915-935.

Dvir, Dov/Lipovetsky, Stanislav/Shenhar, Aaron J. (2003), What is Really Important for Project Success? A Refined, Multivariate, Comprehensive Analysis, in: International Journal of Management and Decision Making, Vol. 4, S. 382-404.

Feldman, Martha S./Pentland, Brian T. (2003), Reconceptualizing Organizational Routines as a Source of Flexibility and Change, in: Administrative Science Quarterly, Vol. 48, S. 94-118.

Freudenberg, Heino (1999), Strategisches Verhalten bei Reorganisationen, Wiesbaden.

Fritz, Wolfgang (2004), Die Erfolgsfaktorenforschung - ein Misserfolg?, in: Die Betriebswirtschaft, 64. Jg., S. 623-625.

Gälweiler, Aloys (2005), Strategische Unternehmensführung, 3. Aufl., Frankfurt/New York.

Gassner, Winfried (1999), Implementierung organisatorischer Veränderungen: Eine mitarbeiterorientierte Perspektive, Wiesbaden.

Goold, Michael/Campbell, Andrew (2002), Designing Effective Organizations, San Francisco.

Haenecke, Henrik (2002), Methodenorientierte Systematisierung der Kritik an der Erfolgsfaktorenforschung, in: Zeitschrift für Betriebswirtschaft, 72. Jg., S. 165-183.

Hair, Joseph F./Anderson, Rolph E./Tatham, Ronald L./Black, William C. (1998), Multivariate Data Analysis, 5. Aufl., Upper Saddle River, NJ.

Hildebrandt, Lutz (1992), Wettbewerbssituation und Unternehmenserfolg. Empirische Analysen, in: Zeitschrift für Betriebswirtschaft, 62. Jg., S. 1069-1084.

Hjelt, Paola (2004), The Secrets of Execution, in: Fortune, Vol. 8, S. 42-43.

Kirsch, Werner/Esser, Werner-Michael/Gabele Eduard (1979), Das Management des geplanten Wandels von Organisationen, Stuttgart. 
Lam, Simon S.K./Chen, Xiao-Ping/Schaubroeck, John (2002), Participative Decision Making and Employee Performance in Different Cultures: The Moderating Effects of Allocentrism/Idiocentrism and Efficacy, in: Academy of Management Review, Vol. 45, S. 905-914.

Latham, Gary P./Kinne, Sydney B. (1974), Improving Job Performance through Training in Goal Setting, in: Journal of Applied Psychology, Vol. 59, S. 187-191.

Lattmann, Charles (1977), Führung durch Zielsetzung, Bern/Stuttgart.

Lawler, Edward E. (1986), High-involvement Management: Participative Strategies for Improving Organizational Performance, San Francisco.

Lechler, Thomas (2005), Projektmanagement. Konzepte zur Einzel- und Multi-Projektführung, in: Albers, Sönkel Gassmann, Oliver (Hrsg.), Handbuch Technologie- und Innovationsmanagement, Wiesbaden, S. 493-510.

Lehner, Franz (1995), Die Erfolgsfaktoren-Analyse in der betrieblichen Informationsverarbeitung, in: Zeitschrift für Betriebswirtschaft, 65 Jg., S. 385-409.

Leidecker, Joel K./Bruno, Albert V. (1984), Identifying and Using Critical Success Factors, in: Long Range Planning, Vol. 17, S. 23-32.

Long, J. Scott (1997), Regression Models for Categorical and Limited Dependent Variables, London.

Malik, Fredmund (1990), Systemisch-evolutionäres Projektmanagement, in: Balck, Henning (Hrsg.), Neuorientierung im Projektmanagement, Köln, S. 77-112.

Malik, Fredmund (2002), Führen Leisten Leben. Wirksames Management für eine neue Zeit, Stuttgart/München.

Marr, Rainer/Steiner, Karin (2004), Projektmanagement, in: Schreyögg, Georg/von Werder, Axel (Hrsg.), Handwörterbuch Unternehmensführung und Organisation, Stuttgart, S. 1196-1208.

Might, Robert J./Fisher, William A. (1985), The Role of Structural Factors in Determining Project Management Success, in: IEEE Transactions Engineering Management, EM-32, Nr. 2, S. 71-77.

Nicolai, Alexander/Kieser, Alfred (2002), Trotz eklatanter Erfolglosigkeit: Die Erfolgsfaktorenforschung weiter auf Erfolgskurs, in: Die Betriebswirtschaft, 62. Jg., S. 579-596.

O'Leary-Kelly, Ann M./Martocchio, Joseph J./Frink, Dwight D. (1994), A Review of the Influence of Group Goals on Group Performance, in: Academy of Management Journal, Vol. 37, S. 1285-1301.

Paper, David, Chang/Ruey-Dang (2005), The State of Business Process Reengineering: a Search for Success Factors, in: Total Quality Management \& Business Excellence, Vol. 16, S. 121-133.

Picot, Arnold/Freudenberg, Heino/Gassner, Winfried (1999) Management von Reorganisationen, Wiesbaden.

Pinto, Jeffrey K./Mantel, Samuel J. (1990), The Causes of Project Failure, in: IEEE Transactions of Engineering Management, EM-37, 4. Jg., S. 269-276.

Pinto, Jeffrey K./Covin, Jeff G. (1989), Critical Factors in Project Implementation: a Comparison of Construction and R\&D projects, in: Technovation, Vol. 9, S. 49-62.

Pümpin, Cuno/Geilinger, Ulrich W. (1988), Strategische Führung: Aufbau strategischer Erfolgspositionen in der Unternehmungspraxis, 2. Aufl., Bern.

Rapoport, Anatol (1992), Weltbilder - Wissen und Glauben: Die systemische Sicht. In Weltbilder - Wissen und Glauben, Aulavorträge, Nr. 55, St. Gallen, S. 3-27.

Rockart, John F. (1979), Chief Executives Define Their Own Data Needs, in: Harvard Business Review, Vol. 57 , S. 81-92.

Rogers, William H. (1993), Regression Standard Errors in Clustered Samples, in: Stata Technical Bulletin, Vol. 13, S. 19-23.

Rüegg-Stürm, Johannes/Young, Monika (2001), Die Bedeutung neuer netzwerkartiger Führungs- und Organisationsformen für die Dynamisierung von Unternehmungen, in: Die Unternehmung, 55. Jg., S. 187-213.

Schmidt, Karsten (2003), Kritische Erfolgsfaktoren im Projektmanagement, in: OrganisationsEntwicklung, No. 3, S. 86-89. 
Schwaninger, Markus (1984), Management-Entwicklung. Ansätze und Erfahrungen, in: Die Unternehmung, 38. Jg., S. 231-243.

Schwaninger, Markus (1989), Integrale Unternehmungsplanung, Frankfurt/New York.

Schwaninger, Markus/Kaiser, Christian (2006), Tragfähiger organisatorischer Wandel, Empirische Studie über die Erfolgsfaktoren bei Organisations- und Veränderungsprojekten, Diskussionsbeitrag Nr. 54, St. Gallen: Institut für Betriebswirtschaft an der Universität St. Gallen.

Schwaninger, Markus/Körner, Markus (2003), Systemisches Projektmanagement. Ein Instrumentarium für komplexe Veränderungs- und Entwicklungsprojekte, in: ZFO-Zeitschrift Führung und Organisation, 72. Jg., S. 75-85.

Schwaninger, Markus/Körner, Markus (2004), Organisationsprojekte managen, 2. Aufl., St. Gallen.

Seibert, Scott E./Silver, Seth R./Randolph, W. Alan. (2004), Taking Empowerment to the Next Level: A Multiple-level Model of Empowerment, Performance, and Satisfaction, in: Academy of Management Journal, Vol. 47, S. 332-349.

Senge, Peter M. (1996), Die fünfte Disziplin: Kunst und Praxis der lernenden Organisation, Stuttgart.

Ulrich, Hans (2001), Gesammelte Schriften, 5 Bände, Bern et al.

Vroom, Victor H. (1964), Work and Motivation, New York et al.

Wagner III, John A. (1994), Participation's Effects on Performance and Satisfaction: A Reconsideration of Research Evidence, in: Academy of Management Review, Vol. 19, S. 312-330.

Williams, Terry M. (1999), The Need for New Paradigms for Complex Projects, in: International Journal of Project Management, Vol. 17, S. 269-273.

Winship, Christopher/Mare, Robert Denis (1984), Regression Models with Ordinal Variables, in: American Sociological Review, Vol. 49, S. 512-525.

\section{Summary}

Empirical Research has hardly dealt with the question of success factors in organizational change. To close that gap, we have used the data from a survey on change projects carried out in eight large corporations. Interviewees were the project managers and additional project staff in organizational change projects. By means of statistical analyses we discerned factors which crucially influence the success of such projects. A number of factors are distinctive for their strong influence on project success: Commitment of decision makers, focus of decisions (content as opposed to expressed interest), clearness of goals, participation, and a holistic approach to project management. Additional analyses were carried out to examine if these results held. This procedure triggered further insights, for example about varying degrees of influence from certain factors. We have not been able to falsify the initial results; on the contrary, they have gained in durability through the additional analyses. Therefore they can be considered as robust. 